\title{
Exploring Opportunistic Access Techniques Using Stochastic Models: Dynamic Spectrum Access Without Sensing
}

\author{
Jad Nasreddine, Janne Riihijärvi, Xia Li, Petri Mähönen \\ Institute for Networked Systems, RWTH Aachen University \\ Kackertstrasse 9, D-52072 Aachen, Germany \\ Email: $\{$ jad, jar, xia, pma\}@inets.rwth-aachen.de
}

\begin{abstract}
In this paper we explore the use of stochastic approaches for opportunistic access based on models of primary user activity. We focus in particular on a scenario where the secondary user does not perform traditional sensing at all, but relies only on statistical information of primary transmissions. Using techniques from the theory of alternating renewal processes we show that, for different primary user constraints, simple statistical transmission schemes exist based on selecting sufficiently low transmission probability for the secondary user. We describe the general framework for deriving the required results, and give a detailed case study assuming that the primary transmitter activity patterns follow exponential distributions. The results for the exponential case show that limited but stable capacity can be achieved by the secondary user for any primary activity level. We also discuss the possible usage scenarios for such statistical approaches, focusing on situations in which traditional sensing is challenging due to high level of dynamics in the environment.
\end{abstract}

\section{INTRODUCTION}

The traditional fixed spectrum assignment policy based on long-term spectrum access licenses for wide geographical areas has reached its limits due to the high increase in the number of wireless technologies, users, and their requirements. This type of assignment lead to the situation where certain portions of the spectrum are highly congested whereas other portions are rarely utilized at some periods of time [1]. Therefore, new approaches have been proposed to solve the problem of inefficient spectrum usage. These approaches range from architectural ones, such as deploying small range cells (e.g., femtocells) [2], to new spectrum access policies, such as Dynamic Spectrum Access (DSA) [3]-[6].

One promising DSA approach is spectrum sharing between primary and secondary networks, where the latter can opportunistically access the licensed spectrum of the former while respecting strict interference constraints [6]-[8]. Most of the work in this context has been focusing on the case where the primary network is a broadcast TV network. In this setting, energy and cyclostationary detection techniques have been studied widely [9]-[12]. However, TV networks have a stable and continuous transmission pattern. Therefore, fast sensing is not required and only spatial characterization of the spectrum opportunities is needed. The other scenario where DSA is interesting is when the primary network is a cellular network with dynamic spectrum usage. This is especially beneficial in the case where the secondary and primary networks belong to the same operator, such as in the case of coexistence between macrocells and femtocells. Other scenarios include, but are not limited to, the case where secondary Machine-toMachine communications or monitoring systems are allowed to opportunistically access the spectrum assigned to a cellular network when a flexible architecture such as DIMSUMnet [13] is used. The cellular operator can be encouraged to share its frequency by the regulator by assigning a set of frequencies to this operator with lower price but with the requirement of sharing these with secondary networks under strict constraints on interference. The problem when considering a cellular primary network is the fast dynamics of the primary network and the presence of several primary transmitters. In this case the traditional sensing-based techniques are not anymore efficient since (1) these techniques have not been studied with the presence of multiple transmitters and power control and (2) they can be very slow compared to the fast dynamics of the traffic of most cellular networks. To solve the problem of multiple transmitters, some location-based approaches [14], [15] were proposed, especially after the adoption of regional database driven techniques by the FCC [16]. Again, these methods will fail to provide good performance in operational networks, especially with primary networks characterized by power control, fast scheduling and short duty cycles, which is unfortunately the case for most cellular networks.

Due to the limitations of sensing- and location-based techniques, we explore in this paper another approach that does not require any sensing or measurement. This will allow secondary network to overcome the problem of primary fast dynamics and multiple transmitters. Our approach is to exploit the information about primary activity patterns and perform statistical transmission with enough low probability to guarantee primary constraints. Therefore, we use alternating renewal theory [17][20] to determine the periods of time where the secondary users can transmit while satisfying statistical primary constraints. Recently, several papers proposed mechanisms based on alternating renewal theory [21], [22] to estimate the best channel to transmit or the available time to transmit after detecting a free channel. These methods are based on the sensing-before-transmit concept, which again yields poor performance in case of highly dynamic primary networks. In this paper, we develop a general framework based on stochastic 
opportunistic access with different types of primary constraints without the need for sensing. Then, we study in detail the case where the primary ON/OFF period durations are modeled with exponentially distributed parameters, which is one of the cases encountered in mobile networks [23].

The rest of the paper is organized as follows. In Section II we introduce the system model, the primary constraint metrics, the general secondary transmission framework and the considered assumptions. In Section III we describe the framework to estimate the primary metrics and determine the transmission periods of the secondary. Furthermore, we discuss in detail the case where the primary active and inactive periods follow exponential distributions. Based on the developed model, we describe in Section IV a practical spectrum access approach. Then we analyze the performance of the proposed method and compare it to sensing- and location-based methods in Section V before we conclude in Section VI.

\section{System Model AND Assumptions}

In this paper, we consider a secondary network that can opportunistically access the licensed spectrum of a primary network under strict constraints guaranteeing the required Quality of Service (QoS) for primary users. Our objective is to find the best secondary activity patterns (i.e., time periods where the secondary node can transmit) over different primary channels leading to the highest possible capacities or lowest transmission delays, while satisfying primary constraints.

The considered primary network comprises $M$ base stations using $N$ channels. The coverage area of its cells is determined by the region where the required QoS is guaranteed with a specific probability and is usually estimated using planning tools. Each base station has an activity pattern on a given channel that can be different from the activity patterns of other base stations or on other channels. The activity patterns are represented by an alternating renewal process of ON (active) and OFF (idle) periods. The durations of the $\mathrm{ON}$ and OFF periods of base station $j$ on channel $k$ are assumed to be independent from each other and represented by independent identically distributed random variables $T_{\mathrm{ON}}^{j, k}$ and $T_{\mathrm{OFF}}^{j, k}$, respectively. These variables follow general distributions with cumulative distribution functions (cdfs) $F_{j, k}$ and $G_{j, k}$, respectively. This model has been found to be realistic for several legacy systems such as GSM and DECT [23].

In the secondary network side, we consider a network with infrastructure including $L$ stationary nodes (i.e. base stations) and a variable number of mobile terminals associated to the different stationary nodes. We assume that the positions of the secondary base stations with respect to the primary base stations are known, for instance, through regional database as was suggested by the FCC [16]. However, this is not a requirement for the proposed approach that can be also implemented without knowing primary base station locations.

\section{A. Primary Constraints}

The objective of the proposed approach is to enable secondary users to access licensed spectrum of a legacy cellular network. The latter has normally strict policies on guaranteeing the desired QoS for its users and will not allow any significant degradation of this QoS. Therefore, cellular networks will allow opportunistic access only when they can still control and guarantee such requirements for their users. Hence, several performance metrics can be defined based on the type of network and application. In this paper we consider the following metrics without limiting the developed framework that can be extended to take into account other metrics ${ }^{1}$ as well:

- $\theta$ : Average fraction of time during which a primary transmission is interfered by secondary activity:

$$
\theta=\frac{E\left(T_{\mathrm{ON}}^{j, k} \mid \mathrm{A} \text { secondary is active }\right)}{E\left(T_{\mathrm{ON}}^{j, k}\right)},
$$

where $E\left(T_{\mathrm{ON}}^{j, k}\right)$ and $E\left(T_{\mathrm{ON}}^{j, k} \mid \mathrm{A}\right.$ secondary is active $)$ are the expectation value of the primary ON periods over time for a given cell $j$ and its conditional expectation given that a secondary node is active, respectively. The primary network can be interested that the users in a given cell will only lose data in $\theta \times 100 \%$ of their transmission time. This is the case for applications that are sensitive to bit error rate such as background download. In this case, the primary network requires that $\theta<\theta_{\text {th }}$ in all cells.

- $\rho$ : The ratio between the average number of primary ON periods lost and the average number of the total ON periods. In this case we assume that if an $\mathrm{ON}$ period is partially interfered (i.e., only a part of the transmission is interfered) it will be lost, for instance due to the loss of the preamble. Thus, $\rho$ can be used especially in the case where the ON periods represent whole packets of a user, such as in the case of the uplink of a Wi-Fi network. For this metric the primary network requires that $\rho<\rho_{\text {th }}$.

We define $\mathcal{S}$ as the set of metrics that the primary operator defines to enable access to its spectrum in an opportunistic way. To estimate these metrics, we consider that a primary receiver $j$ is interfered by a secondary node $l$ if the latter transmits and the probability that the received interference by $j$ due to this transmission is higher than given threshold $\iota_{\max }$ exceeds $\varepsilon$. Formally, we can write this condition as

$$
\mathbb{P}\left\{I_{l j}>\iota_{\max }\right\}>\varepsilon,
$$

where $I_{l j}$ is the interference experienced by primary receiver $j$ due to the transmission of secondary node $l$. The channels that can be shared by secondary nodes as well as the values of $\iota_{\text {max }}$ and $\varepsilon$ are determined by the primary planning tools [25].

To encourage primary operators to share their spectrum, we assume that any approximation in the computation of the metrics in set $\mathcal{S}$ is conservative with respect to primary protection. Therefore, we assume that when a primary is interfered, all data transmission during the interference time are discarded and considered to be erroneous. Moreover, the coverage area of any base station is considered to be the disc

\footnotetext{
${ }^{1}$ The metrics based on the average value can be also replaced by a percentile. The required theory can be found in [17], [24].
} 
enclosing the real coverage area. By using proper propagation models and shadowing models, this disc can be determined. The same conservative approach is used to determine the coverage area of secondary base stations. In general cellular activity patterns are not stable over time. However, for limited periods of time-in the order of few hours-the activity pattern can be relatively stable. Thus, the primary network can characterize these periods by the most conservative pattern (i.e, the pattern that is the most sensitive to secondary activity).

\section{B. Secondary Transmission Framework}

Since no sensing is required in our approach, the secondary node will have a period of transmission $\left(\bar{T}_{\mathrm{ON}}\right)$ followed by a period where it is inactive $\left(\bar{T}_{\mathrm{OFF}}\right)$, where $\bar{T}$ refers to the time of secondary nodes. The sum of these two durations forms the frame of secondary node with duration $\bar{T}$. In general, the frame duration can be either fixed or variable depending on the adopted model. As mentioned before, the objective of the secondary is to maximize its capacity or minimize the delay by finding the best activity pattern. The capacity can be maximized by maximizing the ratio $\bar{T}_{\mathrm{ON}} / \bar{T}$, whereas the delay can be minimized by decreasing the value of $\bar{T}$.

The secondary activity pattern can be determined by either the secondary node or the primary network. In the latter case, the primary will send the allowed computed activity pattern to the secondary without revealing any information about its activity pattern. This approach is suitable when the primary network is not willing to reveal any information about its activity patterns. In the other case, the primary network has to share its activity patterns. This can be possible in case, for instance, that the secondary is a femtocell network controlled by the same primary operator and where the risks are lower to access the sensitive information of the operator. This mode will allow the secondary node to find better solutions, since in the other mode the pattern is computed in a generic way without taking into account the specific characteristics of each secondary node. In order to enable such type of information exchange we assume that there is a minimum cooperation between primary and secondary networks. This is not only beneficial for secondary networks that will be allowed to use the spectrum but also to primary networks; if the secondary network is owned by the same primary operator (e.g., femtocell case), the operator would be interested in increasing the performance of the whole system, including secondary users. Otherwise, the secondary should pay a price for accessing the primary spectrum, based on the type of required QoS.

Different types of activity patterns can be designed for the secondary network. In general we can divide these patterns into two groups: stochastic and periodic. In stochastic patterns, secondary node $l$ will have a policy to be active during a period $\bar{T}_{\mathrm{ON}}$ and inactive during a period $\bar{T}_{\mathrm{OFF}}$ using channel $k$, which follow specific distributions represented by their CDFs $\bar{F}_{l, k}$ and $\bar{G}_{l, k}$. The type of the distributions and their parameters should be computed using $F_{j, k}$ and $G_{j, k}$ for each possible interfered primary cell $j$. The periodic transmission is a special case of the stochastic transmission, where $\bar{T}_{\mathrm{ON}}$ and $\bar{T}_{\text {OFF }}$ are constant values. In this paper we consider the periodic transmission since (1) it contains less variables and it is easier to find the optimal solutions, and (2) it provides more predictable behavior of the secondary activity. However, it is possible to extend the model presented here to the general case by using the approach developed in [20] for the case of several simultaneous alternating renewal processes.

Secondary transmitters are assumed to be cooperative in terms of scheduling the use of primary channels to guarantee that the primary constraints are preserved; the secondary nodes are only allowed to transmit during the scheduled ON periods. Since the secondary ON and OFF periods on each channel are scheduled on a relatively long time scale (e.g., several minutes or hours), synchronization between the different secondary transmitters is possible. This constraint can be relaxed for distant secondary node clusters, where the interference from one cluster to a given primary receiver is negligible whenever the interference from the other cluster is higher than $\iota_{\max }$.

\section{Evaluation of Primary Metrics AND SECONDARY ACTIVITY PATTERN}

In order to evaluate $\theta$ and $\rho$, two main parameters have to be evaluated: the average duration of primary ON periods $\widehat{\theta}(\tau)$ and the average number of ON periods $\widehat{\rho}(\tau)$ during time $\tau$. In this section we consider one channel and one primary cell. Thus, we omit subscripts $j$ and $k$ for the distribution parameters, and the primary metrics can be written as

$$
\begin{aligned}
& \theta=\frac{\widehat{\theta}\left(\bar{T}_{\mathrm{ON}}\right)}{\widehat{\theta}(\bar{T})}, \\
& \rho=\frac{\widehat{\rho}\left(\bar{T}_{\mathrm{ON}}\right)}{\widehat{\rho}(\bar{T})} .
\end{aligned}
$$

In the following, the ON and OFF periods are labeled by states 1 and 0 , respectively. We also denote by $w^{*}(s)$ the Laplace-Stieltjes transform of function $w(t)$ :

$$
w^{*}(s)=\int_{0}^{\infty} e^{-s u} d w(u) .
$$

According to alternating renewal theory, the conditional average number of ON periods $R_{i}(\tau)$ in the interval $[t, t+\tau]$, if the system was in state $i \in\{0,1\}$ at time $t$ is given by [18]

$$
\begin{aligned}
& R_{1}(\tau)=\sum_{n=1}^{\infty} F^{(n)} * G^{(n)}(\tau), \\
& R_{0}(\tau)=\sum_{n=0}^{\infty} F^{(n)} * G^{(n+1)}(\tau) .
\end{aligned}
$$

where $F^{(n)}(\tau)$ and $G^{(n)}(\tau)$ are the $n$-fold convolutions of $F(\tau)$ and $G(\tau)$ with themselves, and $F * G(\tau)$ stands for the convolution of the two functions $F(\tau)$ and $G(\tau)$ of nonnegative variable $\tau$ :

$$
F * G(\tau)=\int_{0}^{\tau} F(\tau-t) d G(t) .
$$


Using the Laplace-Stieltjes transformation, we can write [17]

$$
\begin{aligned}
& R_{1}^{*}(s)=\frac{1-F^{*}(s)}{1-F^{*}(s) G^{*}(s)}, \\
& R_{0}^{*}(s)=\frac{1-F^{*}(s)}{1-F^{*}(s) G^{*}(s)} G^{*}(s) .
\end{aligned}
$$

It should be noted that in case the system is $\mathrm{ON}$ at time $t$, the first ON period is not counted in (9). Thus, in our computation of $\rho(\tau)$ we use $R_{1}(\tau)+1$. Moreover, the conditional average duration of ON periods $\widehat{\theta}_{i}(\tau)$ in the time interval $[t, t+\tau]$ if the system was at state $i \in\{0,1\}$ at time 0 is given by [24]

$$
\widehat{\theta}_{i}(\tau)=\int_{0}^{\tau} A_{i}(u) d u,
$$

where $A_{i}(u)$ represents the point availability at time $u$, which is the probability that the system is $\mathrm{ON}$ at time $u$ conditional on the initial state at time $t$. This probability is given by [18]

$$
\begin{aligned}
& A_{1}(u)=1-F(u)+(1-F) * R_{1}(u), \\
& A_{0}(u)=1-G(u)+(1-G) * R_{1}(u) .
\end{aligned}
$$

The Laplace-Stieltjes transform of the point availability conditional on the initial state is given by [17]

$$
\begin{aligned}
& A_{1}^{*}(s)=\frac{F^{*}(s) G^{*}(s)}{1-F^{*}(s) G^{*}(s)}, \\
& A_{0}^{*}(s)=\frac{G^{*}(s)}{1-F^{*}(s) G^{*}(s)} .
\end{aligned}
$$

For a system that has been running for a long time, the point availability at time $t$ conditional on the state at time 0 can be written as a function of $\lambda$ and $\mu$, which are the inverses of the averages of the ON and OFF period durations [18]:

$$
A=\lim _{t \rightarrow \infty} A_{1}(t)=\lim _{t \rightarrow \infty} A_{0}(t)=\mu /(\lambda+\mu) .
$$

From the law of total probability, we can write using (16)

$$
\begin{aligned}
& \widehat{\theta}(\tau)=\widehat{\theta}_{1}(\tau) A+\widehat{\theta}_{0}(\tau)(1-A), \\
& \widehat{\rho}(\tau)=\left[R_{1}(\tau)+1\right] A+R_{0}(\tau)[1-A] .
\end{aligned}
$$

The ON-OFF periods in wireless communication can follow different types of distributions such as exponential, lognormal, Pareto and Erlang distributions. With the exception of the exponential distribution, it is difficult to derive closed form expressions for $\widehat{\theta}$ and $\widehat{\rho}$. However, numerical evaluation can be done by appropriate extensions of the Cléroux-McConalogue algorithm [26] to evaluate the convolutions. In particular, numerical evaluation of these two metrics have been presented in [19] for Weibull and log-normal distributions. In the following we determine the closed form expressions for $\widehat{\theta}$ and $\widehat{\rho}$ in the the case of exponential distribution.

\section{A. Case Study: Exponential Distribution}

If $F$ and $G$ follow exponential distributions with parameters $\lambda$ and $\mu$, we can easily obtain [17]

$$
\begin{aligned}
\widehat{\theta}_{0}(\tau)= & -\frac{\mu}{(\lambda+\mu)^{2}}\left[1-\exp ^{(\lambda+\mu) \tau}\right]+\frac{\mu \tau}{\lambda+\mu}, \\
\widehat{\theta}_{1}(\tau)= & \frac{\lambda}{(\lambda+\mu)^{2}}\left[1-\exp ^{(\lambda+\mu) \tau}\right]+\frac{\mu \tau}{\lambda+\mu}, \\
R_{0}(\tau)= & -\frac{\mu^{2}}{(\lambda+\mu)^{2}}\left[1-\exp ^{(\lambda+\mu) \tau}\right]+\frac{\mu^{2}}{(\lambda+\mu)^{2}} \\
& +\frac{\lambda}{(\lambda+\mu)^{2}} \tau, \\
R_{1}(\tau)= & \frac{\mu \lambda}{(\lambda+\mu)^{2}}\left[1-\exp ^{(\lambda+\mu) \tau}\right]+\frac{-\mu \lambda}{(\lambda+\mu)^{2}} \\
& +\frac{\lambda}{(\lambda+\mu)^{2}} \tau+1 .
\end{aligned}
$$

By combining (17) and (18) with (19)-(22), we obtain

$$
\begin{aligned}
& \widehat{\theta}(\tau)=\frac{\mu}{\lambda+\mu} \tau, \\
& \widehat{\rho}(\tau)=\frac{\lambda \mu}{\lambda+\mu} \tau+\frac{\mu}{\lambda+\mu} .
\end{aligned}
$$

Finally, we can write the primary metrics for the exponential case using (3), (4), (23) and (24) as

$$
\begin{aligned}
& \theta=\frac{\bar{T}_{\mathrm{ON}}}{\bar{T}}, \\
& \rho=\frac{\lambda \bar{T}_{\mathrm{ON}}+1}{\lambda \bar{T}+1} .
\end{aligned}
$$

It is interesting to see that $\theta$ is independent of the primary activity and $\rho$ depends only on the distribution of primary ON periods.

\section{B. Estimation of $\bar{T}_{O N}$ and $\bar{T}$ for the Exponential Case}

The above equations define the relation between the secondary activity pattern and primary performance metrics. For a specific threshold $\theta_{\text {th }}$ of $\theta$, all values of $\bar{T}_{\mathrm{ON}}$ and $\bar{T}_{\mathrm{OFF}}$ are allowed if they can meet condition (25). This case is suitable especially for real time applications where short delays in transmission are needed since the secondary node can define its transmission periods without any constraints. The maximum achievable $\bar{T}_{\mathrm{ON}} / \bar{T}$ is a constant and equal to $\theta_{\text {th }}$. However, this is not the case for $\rho$ since the values of the durations should be always positive. Therefore, when considering a threshold $\rho_{\text {th }}$ the secondary transmission period should satisfy the following condition:

$$
\bar{T}>\left(\frac{1-\rho_{\mathrm{th}}}{\rho_{\mathrm{th}}}\right) \frac{1}{\lambda} .
$$

This means that the OFF duration of the secondary node may be much higher than the average duration of the ON periods of the primary. This can be very limiting for the case where the secondary wants to have real time communications such as voice. Moreover, from (26), we can write

$$
\frac{\bar{T}_{\mathrm{ON}}}{\bar{T}}=\rho_{\mathrm{th}}-\frac{1-\rho_{\mathrm{th}}}{\lambda \bar{T}},
$$

which is always lower than the value obtained if the same threshold is used for metric $\theta$. Furthermore, it is an increasing function on $\bar{T}$. Therefore the secondary node should make a 


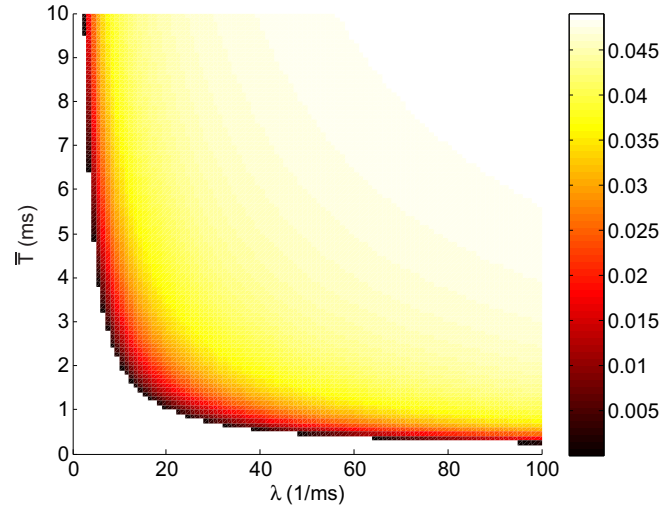

Fig. 1. Variation of the ratio $\bar{T}_{\mathrm{ON}} / \bar{T}$ as a function of $\lambda$ and $\bar{T}$ when $\rho_{\text {th }}=0.05$. The white region in the left corner is the zone where the secondary is forbidden to transmit determined using (27).

tradeoff between increasing its data rate and decreasing delay transmission. An illustrative example of the achievable ratio as a function of $\lambda$ and $\bar{T}$ when $\rho_{\text {th }}=0.05$ is depicted in Fig. 1 . In this case if we want to limit $\bar{T}$ to $10 \mathrm{~ms}$, the secondary can be active only when $\lambda$ is higher than $10 \mathrm{~ms}^{-1}$, meaning that the average primary ON duration should be lower than $0.1 \mathrm{~ms}$.

\section{SPECTRUM ACCESS APPROACH}

For brevity we only consider that the secondary objective is to maximize $\bar{T}_{\mathrm{ON}} / \bar{T}$ in the following. Without the need to do any sensing, the proposed DSA approach based on stochastic models can find the values of $\bar{T}_{\mathrm{ON}}$ and $\bar{T}$ that can be used by a secondary node to transmit on channel $k$ based on the knowledge of $F_{j, k}$ and $G_{j, k}$ for all potentially interfered primary cells. The approach is divided into two main steps for each channel:

1) Find the potentially interfered primary cells,

2) Determine the values of $\bar{T}_{\mathrm{ON}}$ and $\bar{T}$.

\section{A. Determination of the Potentially Interfered Cells}

As discussed in Section II-A, a primary transmission is interfered by secondary activity if condition (2) is met. Let us consider that the secondary node transmits with fixed power $P$ and assume a log-normal distributed shadowing factor with standard deviation $\sigma$. Then, condition (2) can be rewritten as a condition on the distance $d_{s j}$ separating the secondary transmitter $s$ and the primary receiver $j: d_{s j}<d_{\text {th }}$ [14] where

$$
d_{\mathrm{th}}=\mathcal{P}_{s j}^{-1}\left[P-\iota_{\max }+\sigma \sqrt{2} \operatorname{erf}^{-1}(1-2 \varepsilon)\right] .
$$

In (29) $\operatorname{erf}^{-1}$ is the inverse of the error function and $\mathcal{P}_{s j}^{-1}$ is the inverse of the distance dependent path loss function.

In order to estimate $d_{s j}$ we need to determine the position of the primary receiver and secondary transmitter. For each transceiver we have two distinct cases. The transceiver can be either a Mobile Terminal (MT) or a Base Station (BS). If it is a BS, then its position is known. Otherwise, the worst case position is considered, which is the closest position to the secondary transmitter (resp. primary receiver) on the circle enclosing the coverage area of the primary (resp. secondary) base station. Hence, we can write

$$
d_{s j}= \begin{cases}D_{s j} & \text { if two BSs } \\ \max \left\{0, D_{s j}-R_{s}\right\} & \text { if primary BS \& secondary MT } \\ \max \left\{0, \Delta_{s j}\right\} & \text { if primary MT \& secondary BS } \\ \max \left\{0, \Delta_{s j}-R_{s}\right\} & \text { if two MTs }\end{cases}
$$

where $R_{s}$ and $R_{j}$ are the radii of the circles enclosing the coverage areas of the secondary and primary base stations respectively, $D_{j}$ is the distance separating the two base stations, and $\Delta_{s j}=D_{s j}-R_{j}$. Once the distances towards the neighboring cells are computed, condition (29) is verified for all of them. The set of potentially interfered primary cells $\xi$ is then determined as follows:

$$
\xi=\left\{j \in\{1,2, \ldots, M\} \mid d_{s j}<d_{\text {th }}\right\} .
$$

\section{B. Computation of $\bar{T}_{O N}$ and $\bar{T}$}

Let us assume that the primary operator wants to have a constraint on $s$ metrics from set $\overline{\mathcal{S}}=\left\{\kappa_{i} \mid \kappa_{i} \in \mathcal{S}, i=\{1, \ldots, s\}\right\}$ that can include any of the metrics presented in Section II-A or any other possible ones. In our approach, the procedure to compute the activity pattern satisfying primary constraints and maximizing $\bar{T}_{\mathrm{ON}} / \bar{T}$ is divided into three phases.

The first phase aims at finding the secondary activity pattern corresponding to each potentially interfered cell; for each base station $j$ in set $\xi, \bar{T}_{\mathrm{ON}}^{(j)}$ and $\bar{T}^{(j)}$ for a given channel $k$ are computed using the equations of Section III and the functions $F_{j, k}$ and $G_{j, k}$. The second phase uses the computed values of $\bar{T}_{\mathrm{ON}}^{(j)}$ and $\bar{T}^{(j)}$ to reevaluate all metrics $\kappa_{i}$ for each base station except base station $j$ which was evaluated in the previous phase. The obtained values are denoted $\kappa_{i}^{(j)}$. In the third phase, the procedure chooses the activity pattern corresponding to cell $j$ determined as follows

$$
j=\arg \max _{j \in \xi}\left\{\frac{\bar{T}_{\mathrm{ON}}^{(j)}}{\bar{T}^{(j)}} \mid \kappa_{i}^{(j)}<\kappa_{i, \mathrm{th}} \forall \kappa_{i} \in \overline{\mathcal{S}}\right\} .
$$

\section{Evaluation And Comparison With SEnSing- AND LOCATION-BASED TECHNIQUES}

In this section we shall evaluate the performance of the proposed technique and compare the results with sensing- and location-based techniques.

\section{A. System Model}

We evaluate the three approaches in a simple system of seven hexagonal primary cells, where only the downlink case is considered. Each primary base station is located at the center of the cell and transmitting with a power of $30 \mathrm{dBm}$ in each channel. The primary users of each cell are uniformly distributed inside a disc of radius of $1 \mathrm{~km}$. We consider only one channel in our study and assume that only one primary user can be served on this channel at a given time in each cell. The secondary nodes are considered to be some kind of access points that can be at any place in the network and can transmit with a maximum allowed transmit power of $30 \mathrm{dBm}$. 
TABLE I

CONSTANTS OF THE PROPAGATION MODEL.

\begin{tabular}{llll}
\hline & $\alpha(\mathrm{dB})$ & $\beta(\mathrm{dB})$ & $K(\mathrm{~dB})$ \\
\hline Primary BS $\leftrightarrow$ Secondary node & 37.6 & 21 & 113.2 \\
Secondary node $\leftrightarrow$ Primary terminal & 37.6 & 21 & 122.1 \\
\hline
\end{tabular}

We evaluate the different techniques for 100 positions of the secondary nodes distributed in a uniform manner in the zone covering the whole primary system. We assume that the secondary users served by a node are uniformly distributed inside a circle of radius $100 \mathrm{~m}$. We also assume that the activities of the primary base stations follow an exponential distribution with the same parameters of ON/OFF periods (i.e., $\lambda$ and $\mu$ ). Thus, we can define the duty cycle DC of a base station as the proportion of time where the base station is active and is equal to $\mu /(\lambda+\mu)$. In the simulations, we use the Xia-Bertoni propagation model [27] that is able to take into account all types of propagation losses, such as the one between base stations and the one between mobiles in addition to usual propagation loss between a base station and a mobile. Given a frequency $f$ in $\mathrm{GHz}$ and distance $d_{X Y}$ in $\mathrm{km}$ between transmitter $X$ and receiver $Y$, path loss $L_{X Y}$ is given by

$$
\begin{aligned}
L_{X Y} & =\mathcal{P}_{X Y}\left(d_{X Y}\right) \\
& =K_{X Y}+\beta_{X Y} \log _{10}(f)+\alpha_{X Y} \log _{10}\left(d_{X Y}\right),
\end{aligned}
$$

where $K_{X Y}, \beta_{X Y}$ and $\alpha_{X Y}$ are constants computed using the Xia-Bertoni model. The obtained propagation constants are collected in Table I. We also consider a log-normal shadow fading with zero mean and a standard deviation of $7 \mathrm{~dB}$.

The primary metrics are evaluated for each case and compared to the thresholds. For this we assume that a primary cell is interfered when the received interference due to secondary activity is higher than $\iota_{\max }=-100 \mathrm{dBm}$. We also assume that $\varepsilon, \rho_{\text {th }}$ and $\theta_{\text {th }}$ are all equal to 0.05 . The secondary performance is evaluated in terms of average capacity and allowed transmit power. The capacity is computed as the normalized Shannon capacity in bits/s/Hz, $\mathcal{C}=\log _{2}(1+\mathrm{SINR})$. The SINR is computed considering only the interference from primary network and noise power. All techniques were evaluated for different values of $\lambda$ and the duty cycle. In our technique, the value of $\bar{T}_{\mathrm{ON}}$ is computed from (25) and (26), and the value of $\bar{T}$ can be computed as the minimum value satisfying (27).

\section{B. Sensing- and Location-based Techniques}

Sensing techniques in the presence of multiple transmitters have not been studied heavily. There are some preliminary works in this topic that were proposed in [14], [15]. However, these solutions will give lower results in systems with fast power control, fast scheduling, and fast changes in the ON/OFF periods such as the case of mobile networks. Therefore, we consider instead a perfect mechanism but assuming one transmitter in the multi-transmitter case, which gives an upper bound for sensing-based techniques.

Using this assumption, the sensing technique can detect any OFF period where there is no active base station. Therefore the

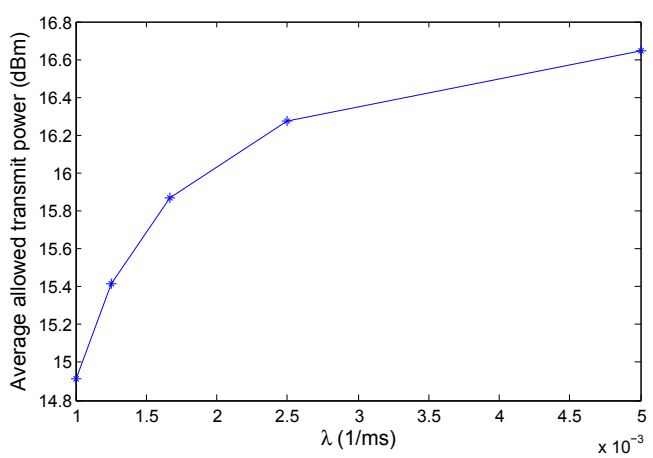

Fig. 2. The average allowed power as a function of $\lambda$ when the secondary uses the stochastic-based method.

OFF periods in this case are the intersection of the OFF periods of the seven cells. We assume that the sensing mechanism can detect an aggregate OFF period with a probability $P_{d}=$ 0.95 , which corresponds to the considered $\varepsilon$ in our algorithm. Moreover, we assume that the probability of false alarm is equal to 0.1 as it is normally considered in the existing works.

The location-based technique is a simplified version of the method proposed in [14]. The main idea is to allow the secondary to transmit with a power that will interfere with the closest base station with a probability $\varepsilon$. In this technique we assume that the positions of the base stations and the secondary nodes are known. In this case, a secondary node $l$ inside the coverage area of an active primary network will not be able to transmit, which is not the case in the proposed approach. To determine the closest active base station, the secondary node compares the received power $S_{l}$ (i.e., the sum of all received powers from active primary base stations) to a location-specific threshold $S_{\text {th }}(l)$ for each base station $l$. This threshold depends on the known distance $D_{l s}$ between $s$ and $l$ and is defined by

$$
S_{\text {th }}(l)=\operatorname{erf}^{-1}(2 \varepsilon-1) \sigma \sqrt{2}+P-\mathcal{P}_{l s}\left(D_{l s}\right),
$$

Using this threshold, the closest active BS is determined by

$$
j=\arg \min _{l}\left\{D_{l s} \mid S_{l} \leq S_{\mathrm{th}}(l)\right\} .
$$

\section{Simulation Results}

We note that all the values of $\rho$ and $\theta$ obtained using the proposed approach were always satisfying the primary conditions. Specifically, in the case of the location- and sensing-based techniques, these values where very low due to the conservative approaches considered.

In the stochastic-based approach, the secondary node transmits with its maximum allowed power during time $\bar{T}_{\mathrm{ON}}$, and it stops any transmission during $\bar{T}_{\text {OFF. }}$. Since the transmission schedule only depends on $\lambda$ as can be seen from (26), the average transmit power over time is always the same for the same value of $\lambda$. Fig. 2 shows that it is also an increasing function of this parameter. This is normal because $\bar{T}_{\mathrm{ON}} / \bar{T}$ is an increasing function of $\lambda$ as can be seen from (28).

In Figures 3 and 4, we show the distributions of the transmit powers as function of the primary duty cycle, when the location- and sensing-based techniques are used, respectively. 


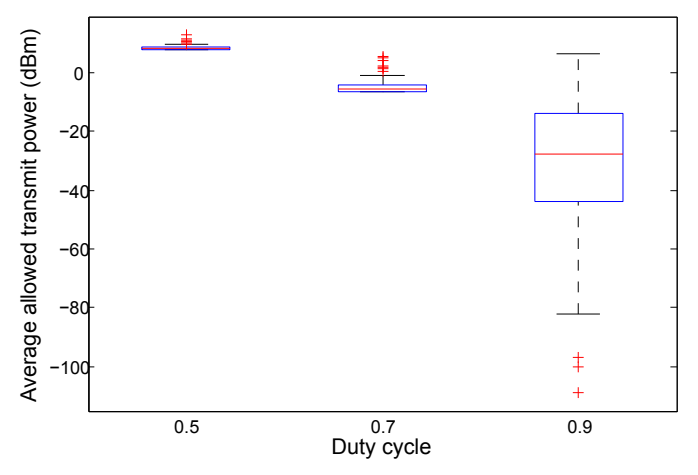

Fig. 3. The average allowed power as a function of the duty cycle when the secondary uses the location-based method for $\lambda=0.005 \mathrm{~ms}^{-1}$.

The figures show that compared to the stochastic technique the transmit powers are very low especially for high duty cycles. It should be noted that when the location-based technique is used, $12 \%$ of the secondary nodes are not allowed to transmit at all. This is the case when the secondary is at the boundary of coverage zones of two or more cells and thus the common OFF periods of the covering base stations are rare for high values of the duty cycle. Moreover, this percentage becomes $97 \%$ when the sensing-based technique is used, since the appearance of common OFF periods between the seven cells is very rare for high duty cycle. In fact, according to (16), the probability of having an OFF period in one base station after long time is $\lambda /(\lambda+\mu)$ which is $1-\mathrm{DC}$, where DC is the duty cycle. Therefore, for 7 cells and a duty cycle of 0.9 the probability of having OFF period in the 7 cells simultaneously is $10^{-7}$.

We shall now study the impact of primary activity on the performance of the secondary users. Fig. 5 shows the distribution of the capacity as a function of the primary duty cycle and for two values of $\lambda$. Although the average transmit power of the stochastic-based technique is very high compared to the two other, the average capacity is relatively low in comparison, especially for low duty cycles. This is due to the fact that in the proposed method, the transmission time is always fixed to the same small value, and the instantaneous capacity is logarithmic function with respect to the transmit power. The figure shows that both sensing-based and locationbased techniques lead to relatively high capacities ranging from 2 to $30 \mathrm{bits} / \mathrm{s} / \mathrm{Hz}$ when the duty cycle is lower than 0.5 . These capacities decrease drastically with the increase in the duty cycle and especially for the sensing-based technique that does not allow any transmission when the duty cycle is higher than 0.5. On the contrary, the stochastic-based approach has a rather stable performance for a given value of $\lambda$ since it does not depend on $\mu$. Even for a duty cycle of 0.9 , this technique allows transmission with median capacity around $0.5 \mathrm{bits} / \mathrm{s} / \mathrm{Hz}$. The decrease in capacity with respect to the duty cycle is due to the interference generated by the primary network. This leads to a decrease in the instantaneous capacity.

It should be noted that the reference techniques are optimal in the sense of detecting all primary opportunities. However, in practice this is not true and the achieved capacity will decrease

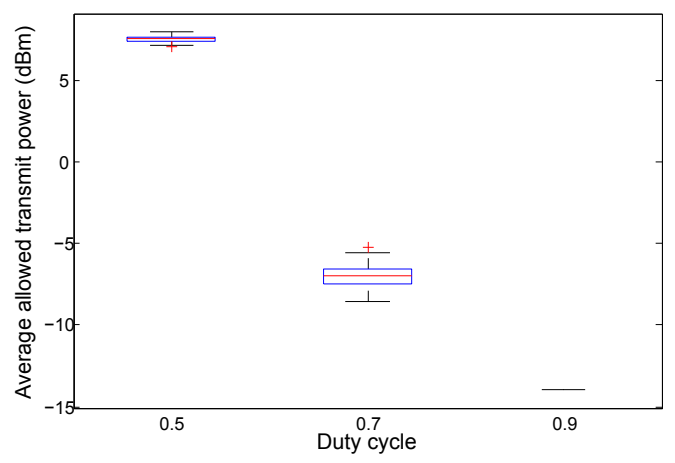

Fig. 4. The average allowed power as a function of the duty cycle when the secondary uses the sensing-based method for $\lambda=0.005 \mathrm{~ms}^{-1}$.

depending on the dynamics of the primary network. This is not the case with the stochastic-based method that, even though it gives low capacity, is able to enable transmission even in the case of high primary activity.

\section{CONCLUSIONS}

In this paper, we have explored a new approach for dynamic spectrum access based on stochastic models. Although the proposed approach does not yield a high capacity, it is suitable especially if the primary activity patterns are very fast where traditional DSA techniques based on sensing and localization fail to allow any transmission. One advantage of the developed approach is its robustness against the fast activity of the primary networks. In contrary to other DSA techniques, it allows a predictable and controllable quality of service. This can be suitable for several applications that do not require high data rate but are more sensitive to jitter and delay.

The results show that the proposed approach cannot perform as well as optimal sensing- and location-based techniques for low duty cycles of primary networks. However, we believe that such techniques are very difficult to implement and stochasticbased approaches can be a good candidate for dynamic spectrum access problems. Furthermore, the new approach reduces power consumption since it does not require sensing. This work is only a first step to explore stochastic-based approaches that can be improved further by considering other statistical aspects of the primary network.

\section{ACKNOWLEDGMENT}

We acknowledge a partial financial support from European Union through FARAMIR project. We also thank the financial support from Deutsche Forschungsgemeinschaft and RWTH Aachen University through UMIC-research centre.

\section{REFERENCES}

[1] "FCC, ET Docket No 03-222 Notice of proposed rule making and order," December 2003.

[2] V. Chandrasekhar, J. Andrews, and A. Gatherer, "Femtocell networks: A survey," IEEE Communications Magazine, vol. 46, no. 9, pp. 59-67, Sept. 2008.

[3] J. A. Hoffmeyer, "Regulatory and standardization aspects of DSA technologies-global requirements and perspective," in the IEEE International Symposium on New Frontiers in Dynamic Spectrum Access Networks (DySPAN 2005), Nov. 2005. 


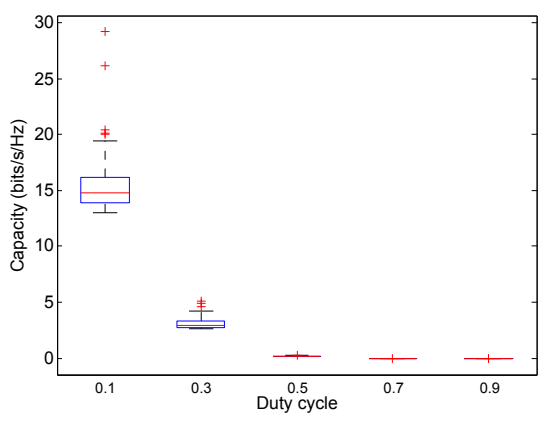

(a)

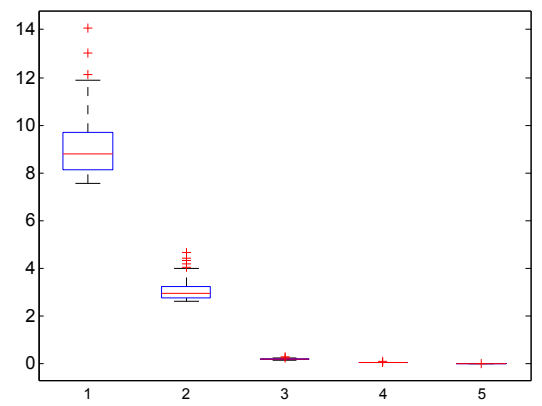

(d)

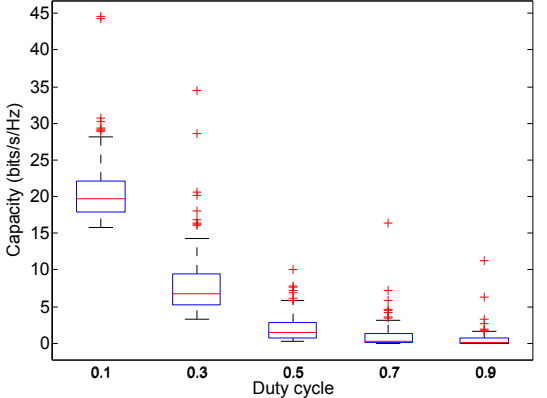

(b)

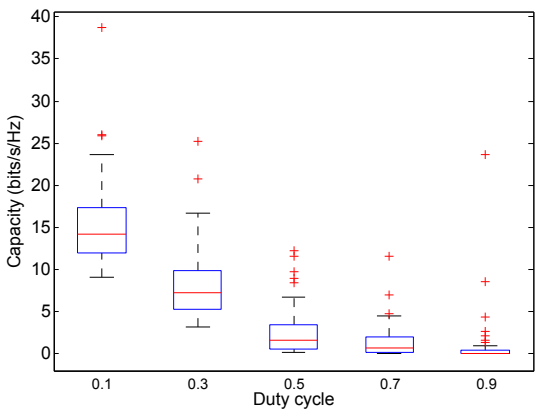

(e)

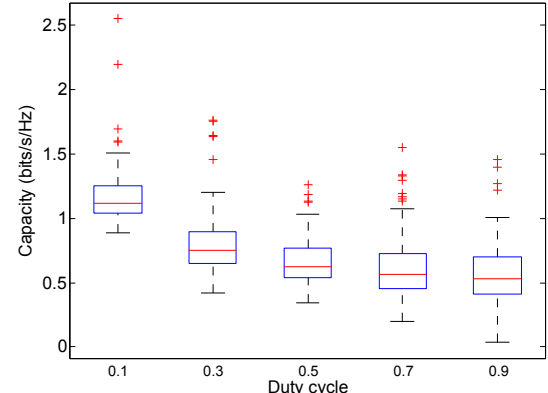

(c)

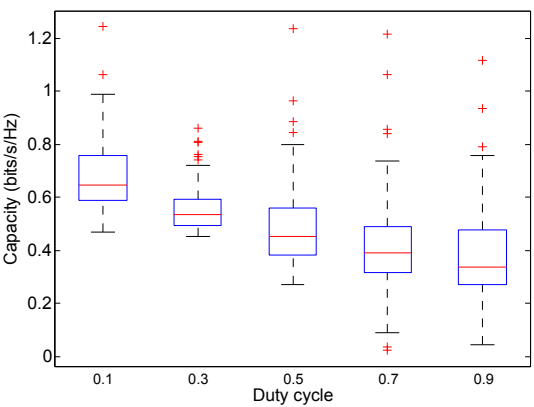

(f)

Fig. 5. The distribution of the capacity as a function of the duty cycle for different values of $\lambda$ when using (a,d) sensing-based, (b,e) location-based and (c,f) stochastic-based techniques. The first row gives the distributions for $\lambda=0.005$ while the second raw for $\lambda=0.001$.

[4] "Spectrum policy task force report," Federal Communications Commission ET Docket 02-135, November 42008.

[5] J. Mitola III, "Cognitive radio for flexible mobile multimedia communications," in IEEE International Workshop on Mobile Multimedia Communications (MoMuC 1999), 1999, pp. $3-10$.

[6] I. F. Akyildiz, W.-Y. Lee, M. C. Vuran, and S. Mohanty, "Next generation/dynamic spectrum access/cognitive radio wireless networks: a survey," Computer Networks: The International Journal of Computer and Telecommunications Networking, vol. 50, no. 13, pp. 2127 - 2159, Sept. 2006.

[7] Q. Zhao and B. M. Sadler, "A survey of dynamic spectrum access," IEEE Signal Processing Magazine, vol. 24, no. 3, pp. 79 -89, May 2007.

[8] R. Tandra, M. Mishra, and A. Sahai, "What is a spectrum hole and what does it take to recognize one?" Proceedings of the IEEE, vol. 97, no. 5, pp. 824-848, 2009.

[9] R. Tandra and A. Sahai, "SNR walls for signal detection," IEEE Journal of Selected Topics in Signal Processing, vol. 2, no. 1, pp. 4-17, 2008.

[10] A. Ghasemi and E. S. Sousa, "Spectrum sensing in cognitive radio networks: the cooperation-processing tradeoff," Wireless Communications and Mobile Computing, vol. 7, no. 9, pp. 1049-1060, 2007.

[11] M. Ghozzi, F. Marx, M. Dohler, and J. Palicot, "Cyclostatilonariltybased test for detection of vacant frequency bands," in the International Conference on Cognitive Radio Oriented Wireless Networks and Communications (CROWNCOM 2006), June 2006, pp. 1-5.

[12] K. W. Choi, W. S. Jeon, and D. G. Jeong, "Sequential detection of cyclostationary signal for cognitive radio systems," Wireless Communications, IEEE Transactions on, vol. 8, no. 9, pp. 4480 -4485, september 2009.

[13] M. M. Buddhikot, P. Kolodzy, S. Miller, K. Ryan, and J. Evans, "DIMSUMnet: New directions in wireless networking using coordinated dynamic spectrum access," in the IEEE International Symposium on a World of Wireless, Mobile and Multimedia Networks (WoWMoM 2005), 2005.

[14] J. Nasreddine, J. Riihijärvi, and P. Mähönen, "Location-based adaptive detection threshold for dynamic spectrum access," in the IEEE International Symposium on New Frontiers in Dynamic Spectrum Access Networks (DySPAN 2010), 2010.

[15] A. O. Nasif and B. L. Mark, "Opportunistic spectrum sharing with multiple cochannel primary transmitters," IEEE Transactions on Wireless Communications, , vol. 8, no. 11, pp. 5702 - 5710, November 2009.

[16] "Second report and order and memorandum opinion and order," in ET Docket No. 04-186 and ET Docket No. 02-380, FCC 08-260, Nov. 2008.

[17] R. E. Barlow and L. C. Hunter, "Reliability analysis of a one-unit system," Operations research, vol. 9, no. 2, pp. 200-208, March-April 1961.

[18] L. A. Baxter, "Availability measures for a two-state system," Journal of Applied Probability, vol. 18, no. 1, pp. pp. 227-235, 1981.

[19] _ "Some remarks on numerical convolution," Communications in statistics. Simulation and computation, vol. 10, no. 3, pp. 281-288, 1981.

[20] _ _ "Availability measures for coherent systems of separately maintained components," Journal of Applied Probability, vol. 20, no. 3, pp. pp. 627-636, Sept. 1983.

[21] H. Kim and K. G. Shin, "Efficient discovery of spectrum opportunities with mac-layer sensing in cognitive radio networks," IEEE Transactions on Mobile Computing, vol. 7, no. 5, pp. 533 -545, May 2008.

[22] M. Sharma, A. Sahoo, and K. D. Nayak, "Model-based opportunistic channel access in dynamic spectrum access networks," in IEEE Global Telecommunications Conference (GLOBECOM 2009), Dec. 2009.

[23] M. Wellens, J. Riihijärvi, and P. Mähönen, "Empirical time and frequency domain models of spectrum use," Physical Communication, vol. 2, no. 1-2, pp. $10-32,2009$.

[24] L. Takás, "Occurrence and coincidence phenomena in case of happenings with arbitrary distribution law of duration," Acta Mathematica Hungarica, vol. 2, pp. 275-298, 1951, 10.1007/BF02020733.

[25] J. Nasreddine, A. Achtzehn, J. Riihijärvi, and P. Mähönen, "Enabling secondary access through robust primary user channel assignment," in IEEE Global Telecommunications Conference (GLOBECOM 2010), Dec. 2010.

[26] R. Cléroux and D. J. McConalogue, "A numerical algorithm for recursively defined convolution integrals involving distribution functions," Management Science, vol. 22, no. 10, pp. 1138-1146, 1976.

[27] L. Maciel, H. Bertoni, and H. Xia, "Unified approach to prediction of propagation over buildings for all ranges of base station antenna height," IEEE transactions on vehicular technology, vol. 42, no. 1, pp. 41-45, Feb. 1993 